\title{
Beauty and Inexhaustibility of Magnetic Resonance
}

\author{
Vadim A. Atsarkin • Valery F. Tarasov • \\ Dmitry A. Tayurskii
}

Published online: 28 October 2014

(C) Springer-Verlag Wien 2014

This issue of Applied Magnetic Resonance is timed to the 70th anniversary of the discovery of electron paramagnetic resonance (EPR) by E.K. Zavoisky in Kazan as early as 1944. In fact, this was the first implementation of magnetic resonance in condensed matter. Two years later, nuclear magnetic resonance was observed and described theoretically by E.M. Purcell, R.V. Pound, and F. Bloch, and soon other kinds of spin resonances such as nuclear quadrupole resonance, ferromagnetic resonance, electron nuclear double resonance, etc. were implemented. All these phenomena based on specific features of electron and nuclear spin dynamics in static and alternating magnetic fields constitute now a vast area of contemporary science and technology.

The readers of Applied Magnetic Resonance are aware of great possibilities of magnetic radiospectroscopy. There is no need to list here its innumerable applications ranging from studies of molecular structures to petroleum prospecting, from spintronics to archaeology, from chemical kinetics to nuclear physics. A true triumph was the invention of magnetic resonance imaging with its broad and fruitful applications in medicine. In the last 70 years, studies related to various magnetic resonance methods gained a constellation of Nobel Prizes.

The apparent inexhaustibility of magnetic resonance methods and applications is really amazing. We presume that it is related to specific beauty of this physical phenomenon which is often (and fairly) called "spin ballet". Sophisticated

\footnotetext{
V. A. Atsarkin $(\square)$

Kotel'nikov Institute of Radio Engineering and Electronics, Russian Academy of Sciences, 125009 Moscow, Russia

e-mail: atsarkin@mail.cplire.ru

V. F. Tarasov

Zavoisky Physical-Technical Institute, Russian Academy of Sciences, 420029 Kazan, Russia

D. A. Tayurskii

Institute of Physics, Kazan Federal University, 420008 Kazan, Russia
} 
manipulations over spin systems performed by modern researchers have no precedent in other fields of science: it suffices to remind the famous experiments with time reversal implemented for the first time by the late Professor J. Waugh. Thus, the spin systems are frequently used as a unique polygon for testing and implementation of the most daring ideas, both in pure physics and technology. A typical example is quantum computing.

Naturally, the papers collected in this issue cannot cover all aspects of magnetic resonance and its applications. Rather, this is a current state of the art of research giving the idea about the typical technical level, most popular directions and hot points. Some bias in favor of strongly correlated and magnetically ordered electron spin systems evidently reflects modern trends. At the same time, bearing in mind the specific character of an anniversary issue, a number of review articles with the historical aspect are included as well.

Note that a considerable part of the issue is composed of papers by Kazan scientists. This seems to be appropriate because Kazan is the birthplace of electron paramagnetic resonance and hosts the celebrated scientific school on the basis of the Kazan Federal University and Zavoisky Physical-Technical Institute, Russian Academy of Sciences.

We hope that the publications presented in this issue will stimulate further progress in magnetic resonance and its applications. 